\title{
Molecular characterization of bla TEM and bla CTX-M in Multi-Drug Resistance $E$. coli isolated from Country Chicken and Country Chicken Eggs in Poultry Farms of Malaysia $^{\dagger}$
}

\author{
Nagaraja Suryadevara 1, Raja Rabiyatun Adawiyah Binti Raja Perdaus 1, Devarani 1," \\ 1 School of bioscience, Faculty of Medicine, Bioscience and Nursing, MAHSA University Bandar Saujana Putra, 41200, \\ Selangor, Malaysia \\ * Correspondence: devarv1022@gmail.com; \\ $\uparrow \quad$ Presented at International e-Conference on Bioengineering for Health and Environment (ICBHE 2020)
}

Received: 5.07.2020; Revised: 10.07.2020; Accepted: 12.07.2020; Published: 15.07.2020

\begin{abstract}
Over the years, multidrug-resistant Escherichia coli has contributed to the development of extended-spectrum beta-lactamase (ESBLs), which evolved primarily from poultry in every corner of the world. The unregulated use of antibiotics commonly administered to poultry products to prevent any subclinical infections that lead to multidrug resistance (MDR) that is due to acquired bacteria resistance. The main aim of this study is to investigate the prevalence in Malaysia of multidrug-resistant, extended-spectrum beta-lactamase (ESBL) producing E.coli from poultry country chicken and country eggs. In several states of Malaysia, fifty samples from country chicken and country chicken eggs were extracted randomly from chosen poultry. The sample was taken from May to June 2019. The samples were tested using traditional microbiological techniques for the presence of $E$. coli. Antibiotics susceptibility test using 5 forms of $\beta$-lactam antibiotics was used using the double-disk diffusion screening, and confirmation of the test is performed by a combination disk diffusion process to establish the strains generating the ESBL. Although, the phenotypic characterization of bla TEM and bla CTXM ESBL was carried out using PCR and SDS methods. Twenty of the fifty samples collected were classified as E.coli (20/50), suggesting 40\%. The results of the distribution of the $\beta$-lactamases genes were reported as bla TEM, bla CTX-M with results of 75\% (3/4), and 100\% (4/4), respectively. The findings indicate a high prevalence of multidrug resistance as the most prevalent of all ESBL genes in ESBL infections with CTX-M genes. Consequently, effective monitoring of MDR infections, in particular resistance to $\beta$-lactamases in poultry chicken, can predict the potential for ESBL infections in humans and animals.
\end{abstract}

Keywords: Multidrug-resistant; E.coli; extended-spectrum beta-lactamase (ESBL); $\beta$-lactam antibiotics; CTX-M genes.

(c) 2020 by the authors. This article is an open-access article distributed under the terms and conditions of the Creative
Commons Attribution (CC BY) license (https://creativecommons.org/licenses/by/4.0/).

Funding

This research received no external funding.

\section{Acknowledgments}

This research has no acknowledgment.

\section{Conflicts of Interest}

The authors declare no conflict of interest.

https://conferenceproceedings.international 